\title{
THE STRUCTURE OF ATOMIC NITROGEN ADSORBED ON $\mathrm{Fe}(100)$
}

\author{
R. IMBIHL, R.J. BEHM and G. ERTL \\ Institut für Physikalische Chemie, Universität München, D-8000 München 2, Fed. Rep. of Germany
}

and

\section{W. MORITZ}

Institut für Kristallographie und Mineralogie, Universität Müchen, D-8000 München 2, Fed. Rep. of Germany

Received 16 July 1982; accepted for publication 2 September 1982

Nitrogen atoms adsorbed on a $\mathrm{Fe}(100)$ surface cause the formation of an ordered $\mathrm{c}(2 \times 2)$ overlayer with coverage 0.5 . A structure analysis was performed by comparing experimental LEED $I-V$ spectra with the results of multiple scattering model calculations. The $\mathrm{N}$ atoms were found to occupy fourfold hollow sites, with their plane $0.27 \AA$ above the plane of the surface $\mathrm{Fe}$ atoms. In addition, nitrogen adsorption causes an expansion of the two topmost Fe layers by $10 \%(=0.14 \AA)$. The minimum $r$-factor for this structure analysis is about 0.2 for a total of 16 beams. The resulting atomic arrangement is similar to that in the (002) plane of bulk $\mathrm{Fe}_{4} \mathrm{~N}$, thus supporting the view of a "surface nitride" and providing a consistent picture of the structural and bonding properties of this surface phase.

\section{Introduction}

Catalytic synthesis of ammonia over iron catalyst proceeds through atomically adsorbed nitrogen (whose formation is the rate-limiting step) [1], and therefore knowledge on the surface structure of this species is of importance for understanding the microscopic reaction steps. Previous LEED investigations [2-4] revealed that on $\mathrm{Fe}(100)$ a simple $\mathrm{c}(2 \times 2)$ structure is formed, while with $\mathrm{Fe}(110)$ or (111) surfaces a series of complicated diffraction patterns was observed which strongly suggested the occurrence of surfacc reconstruction.

It was proposed [3,4] that these surface phases (which were called "surface nitrides") exhibit structures which closely resemble those of the bulk nitride $\mathrm{Fe}_{4} \mathrm{~N}$, even if the latter cannot be formed for thermodynamic reasons. According to this concept the $\mathrm{Fe}(100)-\mathrm{c}(2 \times 2)-\mathrm{N}$ structure should be similar to the (002) plane of $\mathrm{Fe}_{4} \mathrm{~N}$, while with $\mathrm{Fe}(110)$ and (111) no low-index planes of 
$\mathrm{Fe}_{4} \mathrm{~N}$ [5] are compatible without pronounced displacements of iron atoms, thus causing reconstruction.

The present work concerns a structural analysis of the $\mathrm{Fe}(100)-\mathrm{c}(2 \times 2)-\mathrm{N}$ surface phase on the basis of LEED intensity/voltage $(I-V)$ data which will confirm the proposed picture.

\section{Experimental}

The experiments were performed with a standard UHV system (base pressure below $2 \times 10^{-10}$ Torr) which was equipped with a four-grid LEED optics serving also as retarding field analyser for AES. $I-V$ spectra were recorded with a moveable Faraday cup (aperture $14^{\circ}$ ). A system of Helmholtz coils controlled by a magnetic field probe (Förster) was used in order to suppress magnetic stray fields to $\leqslant 1 \mathrm{mOe}$.

The sample was cut from a single crystal rod (purity $99.99 \%$, Metals Research) and oriented to within $1 / 2^{\circ}$. The surface impurities (mainly $\mathrm{C}, \mathrm{O}$ and $S$ ) were removed by prolonged oxidation and sputter/annealing cycles as described earlier [2]. The cleanliness was checked by AES. During the LEED measurements the peak ratios of the $\mathrm{C}(270 \mathrm{eV})$ and $\mathrm{O}(512 \mathrm{eV})$ signals over the $\mathrm{Fe}(650 \mathrm{eV})$ signal could always be kept below 0.05 , indicating impurity concentrations of less than a few percent of a monolayer.

The ordered $c(2 \times 2)$ overlayer of chemisorbed nitrogen atoms was produced by thermal decomposition of $\mathrm{NH}_{3}$ at $670 \mathrm{~K}$ [6] which needs considerably lower gas exposures as if $\mathrm{N}_{2}$ is used [2]. This procedure leaves only nitrogen on the surface since hydrogen desorbs at much lower temperature [6]. The onset of bulk- $\mathrm{Fe}_{4} \mathrm{~N}$ formation occurs, on the other hand, at considerably higher exposure than applied here [7]. Nevertheless part of the nitrogen atoms formed diffuse into the bulk as demonstrated previously by means of isotope exchange experiments [3].

The $c(2 \times 2)$ structure was characterised by an Auger intensity ratio $y_{N}=N$ $(380 \mathrm{eV}) / \mathrm{Fe}(650 \mathrm{eV})=0.6$. The half-order LEED spots were rather sharp and exhibited intensities of about $30 \%$ of the substrate lattice spots.

The LEED measurements were performed after brief heating of the sample to $700 \mathrm{~K}$ (in order to remove adsorbed residual $\mathrm{NH}_{3}$, etc.) and cooling down to room temperature. $I-V$ curves were recorded in steps of $1 \mathrm{eV}$ between 40 and $200 \mathrm{eV}$ for a total of seventeen beams, namely 9 integral-order and 8 fractional-order beams at three different angles of incidence $(\theta=0,6$ and $\left.11.6^{\circ}\right)$. No correction of the energy scale was made for the difference of the work function of the sample $(5.2 \mathrm{eV})$ and the tungsten filament of the LEED gun $(4.5 \mathrm{eV})$ in view of the general uncertainty $(-1 \mathrm{eV})$ of the voltage scale.

Beam profile measurements revealed that at $56 \mathrm{eV}$ only about $50 \%$ of the integral intensity of a beam is collected by the Faraday cup with its small 
aperture $\left(\hat{=} 1^{\circ}\right)$. Since with increasing energy the diffraction spot diameter decreases (as long as it is not limited by the instrumental function) also an increasing fraction of the integral intensity is recorded. As a consequence the relative intensities are somewhat too high at higher electron energies. It was, however, estimated, that this effect is only of very minor influence on the $r$-factor determination, and therefore no correction was made.

\section{Calculation procedures}

A Hartree-Fock-Slater selfconsistent potential was used for $\mathrm{Fe}$ in analogy with previous work $[8-10]$. The nitrogen potential was constructed from a superposition of free atom potentials in a bcc unit cell [11]. LEED intensity calculations were performed using the layer-doubling method [12] for multiple scattering between bulk layers, while the small distance between the $\mathrm{N}$ layer and the topmost $\mathrm{Fe}$ layer required a solution by means of matrix inversion in angular momentum representation within the first two layers. Up to 28 symmetrized beams at normal incidence were involved in the layer-doubling scheme for the models with an adsorption site of fourfold symmetry. A corresponding large number of beams was used for the bridge-site models with p2 $\mathrm{mm}$ symmetry as well as for oblique incidence. The number of phase shifts was reduced to seven, since the calculation for the first layer with three atoms per unit cell required rather long computing times. It was checked that seven phase shifts were sufficient for the energy range up to $200 \mathrm{eV}$; calculations with eight phase shifts yielded only minor differences.

The real part of the inner potential was assumed to be independent of energy. Its optimum value found was $V_{0}=9.5 \mathrm{eV}$, whereby no correction for the work function difference is taken into account. The imaginary part of the inner potential was set equal to $0.85 E^{1 / 3}[\mathrm{eV}]$, which yielded better agreement between theory and experiment than the smaller value of approximately $0.7 E^{1 / 3}[\mathrm{eV}]$ as used by Legg et al. [8] in a study of the clean $\mathrm{Fe}(100)$ surface. No further efforts were made to optimize this as well as other non-structural parameters. The bulk Debye temperature is $\theta_{\mathrm{D}}=467 \mathrm{~K}[13]$ and for the surface layer a value of $400 \mathrm{~K}$ was assumed in order to allow a slightly larger mean square vibrational amplitude for the topmost atoms. Variation of the surface Debye temperature was of only small effect on the calculated $I-V$ spectra, and the results were not significant enough in order to draw any firm conclusion on the actual mean square vibrational amplitudes.

\section{Results}

Before starting the analysis of the overlayer structure, data for the clean $\mathrm{Fe}(100)$ surface were investigated. Both the experimental and the calculated 
results were found to be in excellent agreement with those reported by Legg et al. [8] and are therefore not repeated here. The topmost interlayer spacing is slightly reduced (by about $1.5 \%$ ) with respect to the bulk value: A minimum averaged reliability factor $r=0.15$ was determined for four beams, using the $r$-factor program by Zanazzi and Jona [14].

The formation of the $\mathrm{N}$ overlayer causes not only the appearance of new fractional-order beams, but also affects the $I-V$ spectra from the integral-order beams. As an example fig. 1 compares the $I-V$ data of the $(00)$ beam at $11.6^{\circ}$ angle of incidence for both the clean (b) and nitrogen-covered (a) $\mathrm{Fe}(100)$ surface. For this reason also the $r$-factors for the integral-order beams were included into the structural analysis.

A cubic (100) face exhibits three types of high symmetry adsorption sites, namely the fourfold hollow and "on top" sites, and the twofold bridge site. The latter requires averaging over two domains in order to retain the fourfold symmetry of the $c(2 \times 2)\left(\operatorname{or}(\sqrt{2} \times \sqrt{2}) \mathrm{R} 45^{\circ}\right)$ lattice. Calculations were performed for each of these three models. The atomic radius of $\mathrm{N}$ was allowed to vary betwen 0.5 and $1.0 \AA$, giving rise to corresponding changes in the spacing $d_{\mathrm{N}}$ between the nitrogen and the first iron layer in steps of $0.05 \AA$. The Fe-Fe layer spacing in the bulk is $1.433 \AA$. The distance between the two topmost $\mathrm{Fe}$ layers $d_{\mathrm{Fe}}$ was varied in steps of $0.03 \AA$ between 1.35 and $1.60 \AA$.

Fig. 2 shows a set of experimental data taken at normal incidence together with representative theoretical spectra calculated for the three models indicated. It becomes evident that the best agreement is obtained for the fourfold hollow sites structure as reproduced in fig. 3a. Some calculations were also

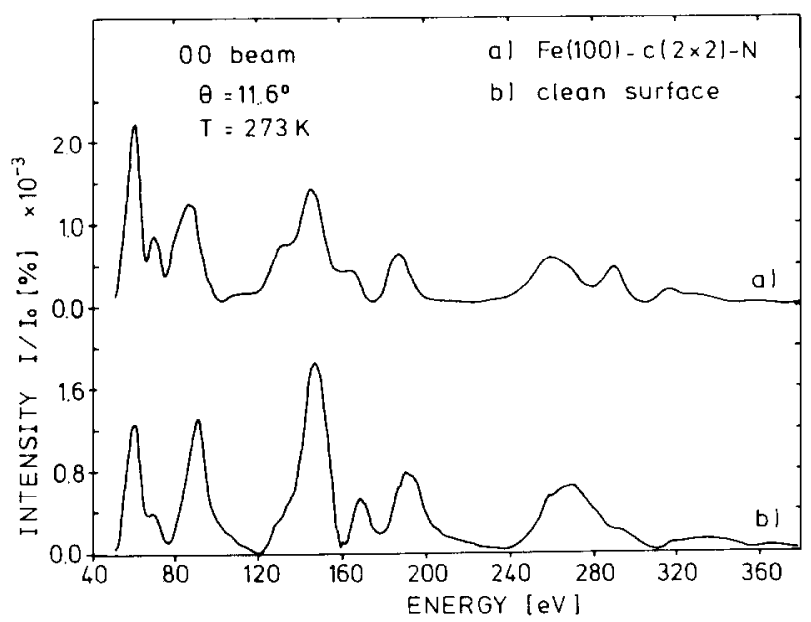

Fig. 1. Intensity-voltage spectra for the $(00)$ beam of the adsorbate covered (a) and clean (b) Fe(100) surface. Angle of incidence $\theta=11.6^{\circ}$. 


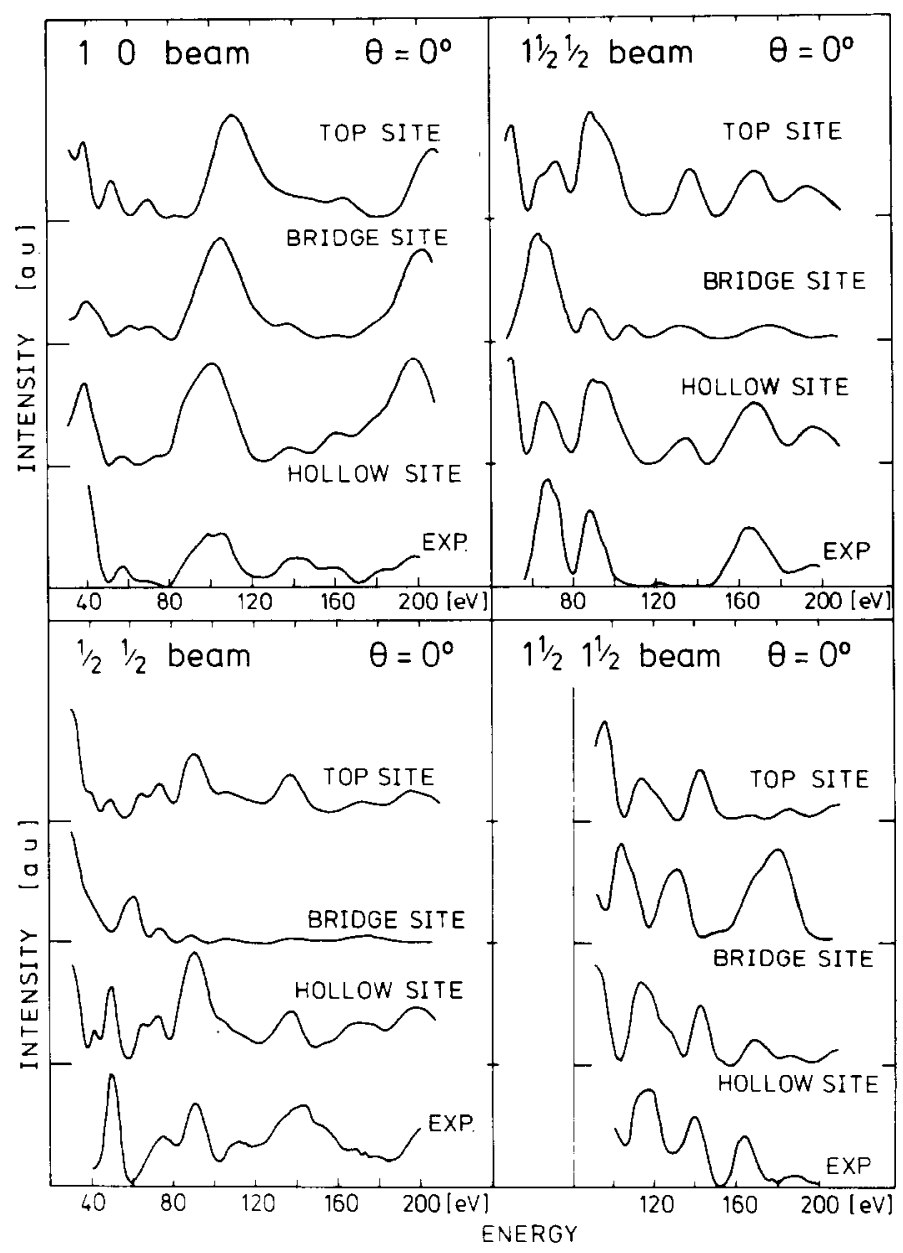

Fig. 2. $I-V$ spectra calculated for $\mathrm{N}$ atoms in the three high-symmetry adsorption sites are compared with the experimental data (normal incidence, $\theta=0^{\circ}$ ).

performed for the "underlayer" model (i.e. where the $\mathrm{N}$ atoms are located below the topmost layer of metal atoms) as derived for the N/Ti(0001) system [15] but the results gave no evidence for such a structure in the present case.

Quantitative structure analysis was now performed for the fourfold hollow site model of fig. 3a. Figs. 4 and 5 compare representative theoretical spectra calculated for varying $d_{\mathrm{N}}$ and $d_{\mathrm{Fe}}$, respectively, with the corresponding experimental data. Only those results are reproduced which revealed to be sensitive to small variations of the structural parameters. It turned out that $d_{N}$ affected 


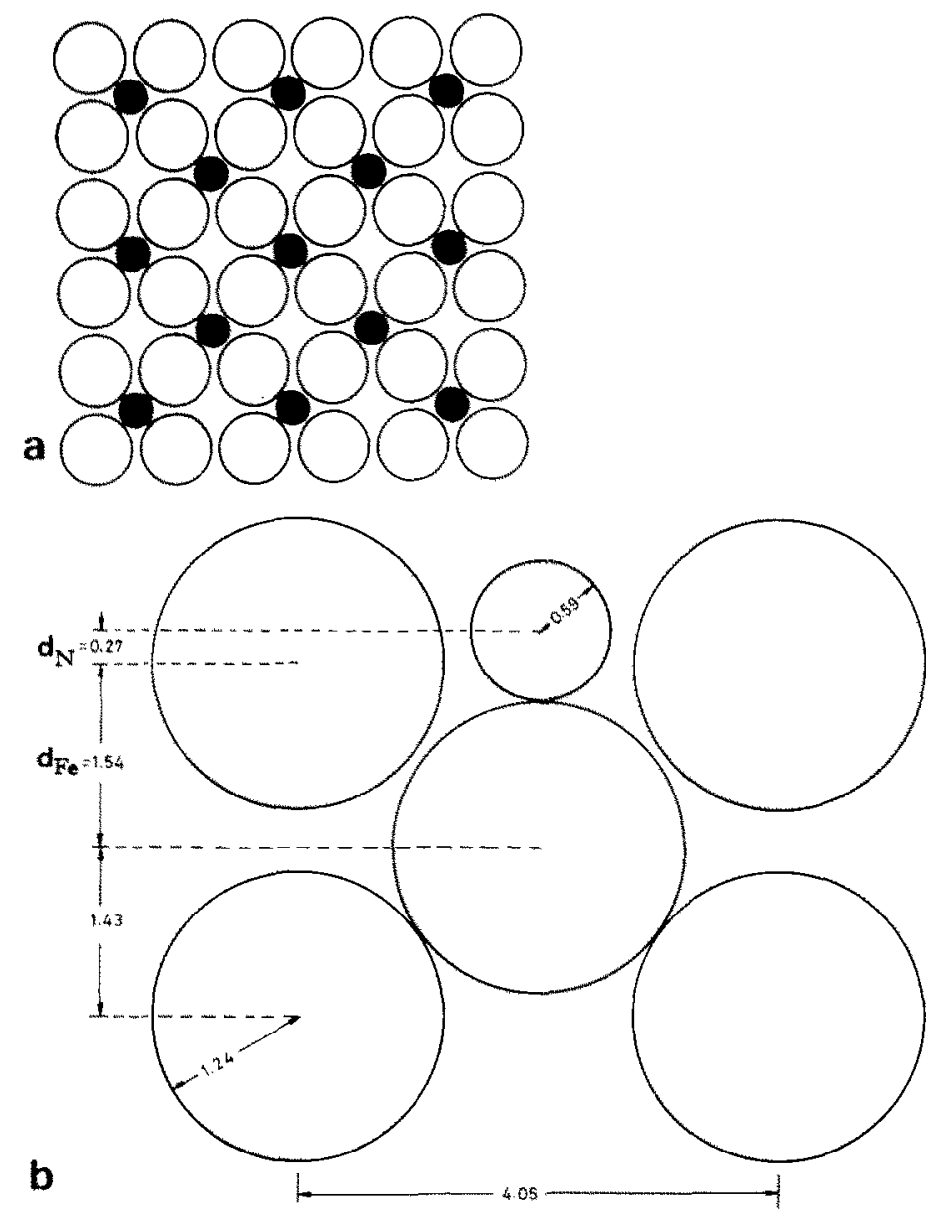

Fig. 3. Hard-sphere model of the Fe(100)-o( $2 \times 2)-\mathrm{N}$ structure. (a) Top view: open circles, Fe atoms; full circles, $N$ atoms in the fourfold hollow sites. (b) Cross section atong the (110) plane. Distances are given in $\AA$.

mainly the fractional-order beams, but that the integral-order beams, on the other hand, were essentially influenced by variations of $d_{\mathrm{Fe}}$.

The best quantitative agreement between experiment and theory was sought by using the $r$-factor concept introduced by Zanazzi and Jona [14]. Variation of both $d_{\mathrm{N}}$ and $d_{\mathrm{Fe}}$ changes also the bond length between the $\mathrm{N}$ atoms and the underlying $\mathrm{Fe}$ atom (see fig. $3 \mathrm{~b}$ ). As a consequence the minima of the $r$-factor curves are shifted systematically to lower values of $d_{\mathrm{re}}$ if $d_{\mathrm{N}}$ is increased. This 


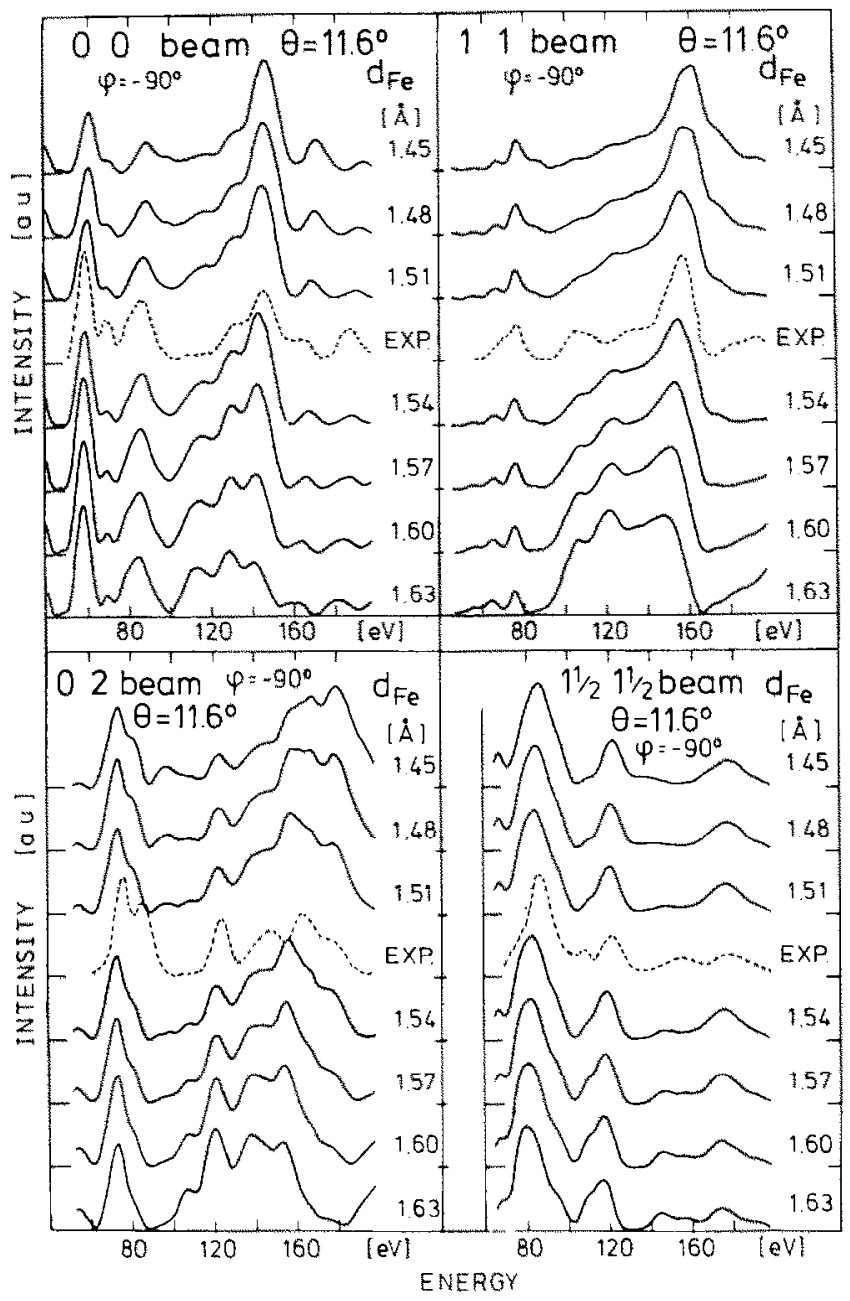

Fig. 4. Comparison between experimental spectra (dotted lines) and theoretical data (full lines) calculated for various interlayer spacings $d_{\mathrm{Fe}}$ between the topmost two planes of $\mathrm{Fe}$ atoms. The distance $d_{\mathrm{N}}$ between the plane of $\mathrm{N}$ atoms and the first layer of Fe atoms was kept fixed at $0.25 \AA$.

becomes evident from fig: 6 showing data for several fractional order beams. The expansion of the Fe-Fe layer spacing at the surface becomes evident from fig. 7 which shows $r$-curves for various integral order beams as a function of $d_{\mathrm{Fe}}$ for the optimum $d_{\mathrm{N}}(=0.25 \AA)$. The minimum ranges between 1.51 and $1.57 \AA$.

The optimum values for the structural parameters were found, as usually, by 


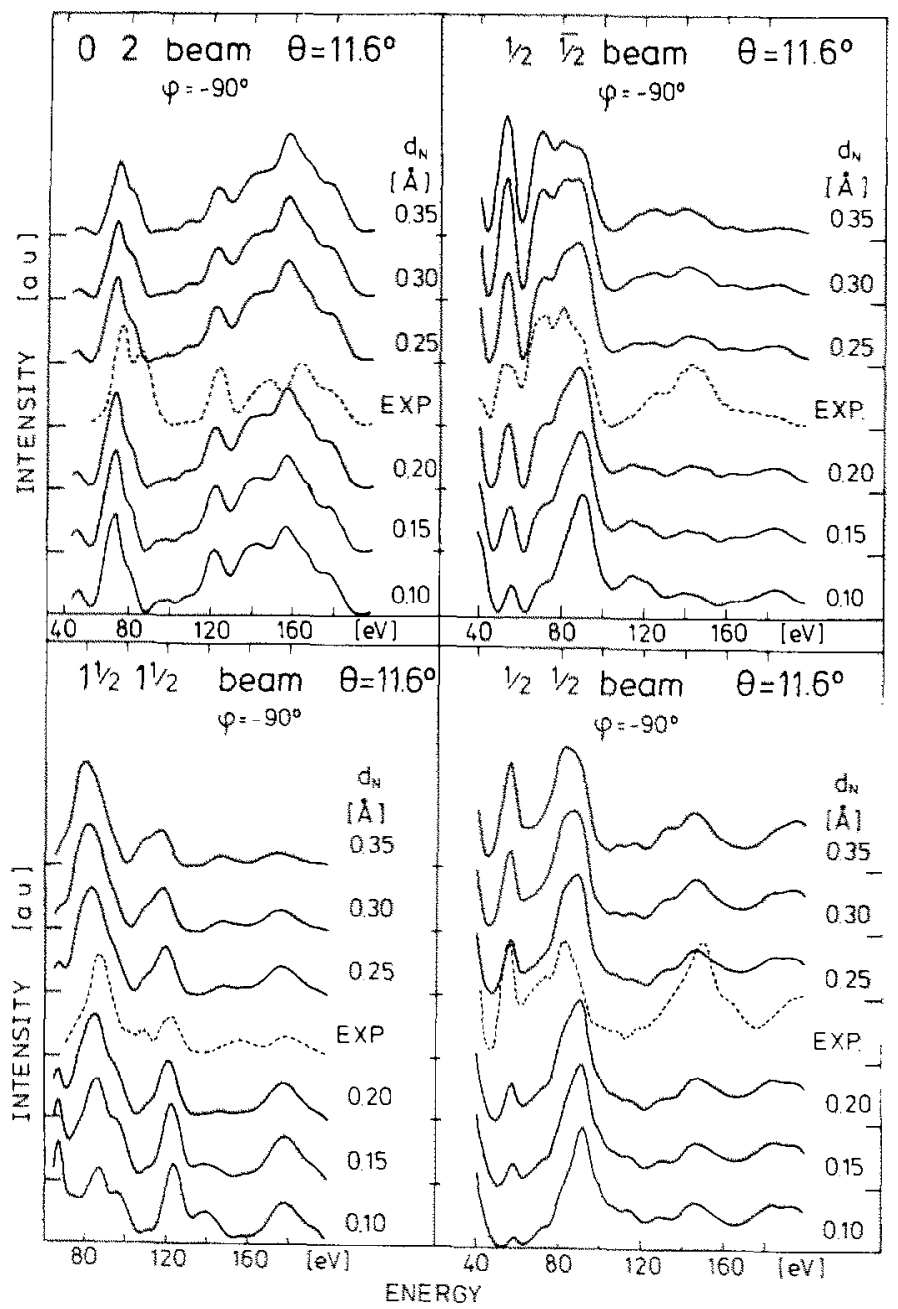

Fig. 5. Comparison between experimental spectra (dotted lines) and theoretical data (full lines) calculated for various values of $d_{\mathrm{N}}: d_{\mathrm{Fe}}$ was kept fixed at $1.54 \mathrm{~A}$.

averaging over all available $r$-factor curves. The average was taken ove 16 beams, namely 6 beams at normal incidence and 10 beams at $\theta=11.6^{\circ}$, $\varphi=0^{\circ}$. The resulting data are shown in fig. 8 as variation of $r$ with $d_{\mathrm{Fe}}$ at different values of $d_{\mathrm{N}}$. The minima are relatively flat which is mainly caused by the fact that a series of beams remains nearly unaffected by relatively large variations of the interlayer distances. The best fit between theory and experiment is obtained for $d_{N}$ between 0.25 and $0.30 \AA$, yielding an optimum value 


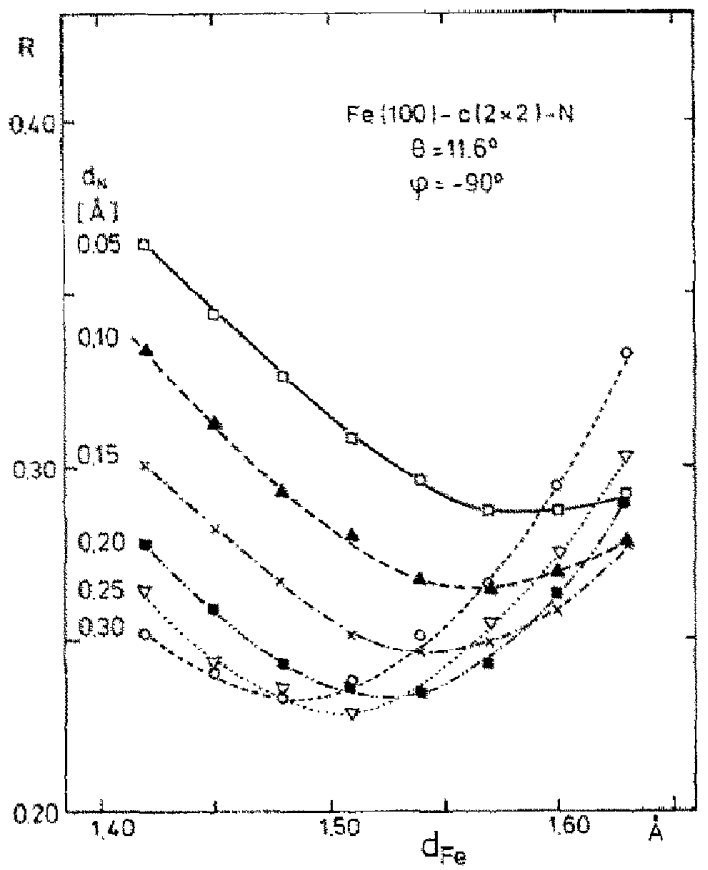

Fig. 6. F-Faster averaged over 5 half-order beams as a function of $d_{\mathrm{F}}$. Paraneter is $d_{\mathrm{m}} \mathrm{d}$

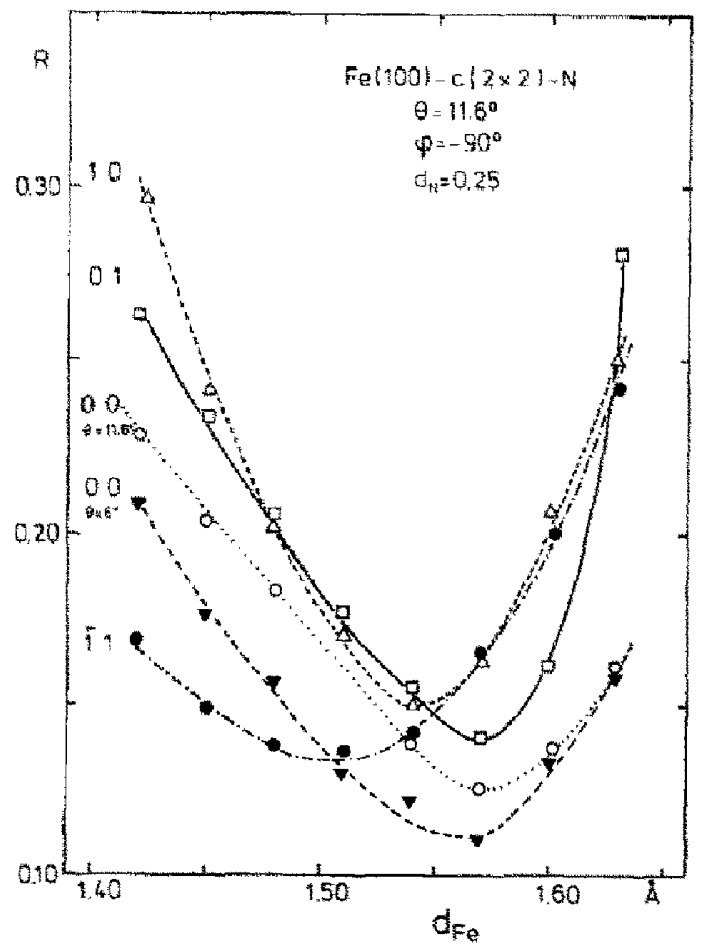

Fig. 7. r-Factor for 5 integralorder beams as a function of $d_{F_{2}}: d_{\mathrm{N}}=0.25 \AA$ was kept constant. 


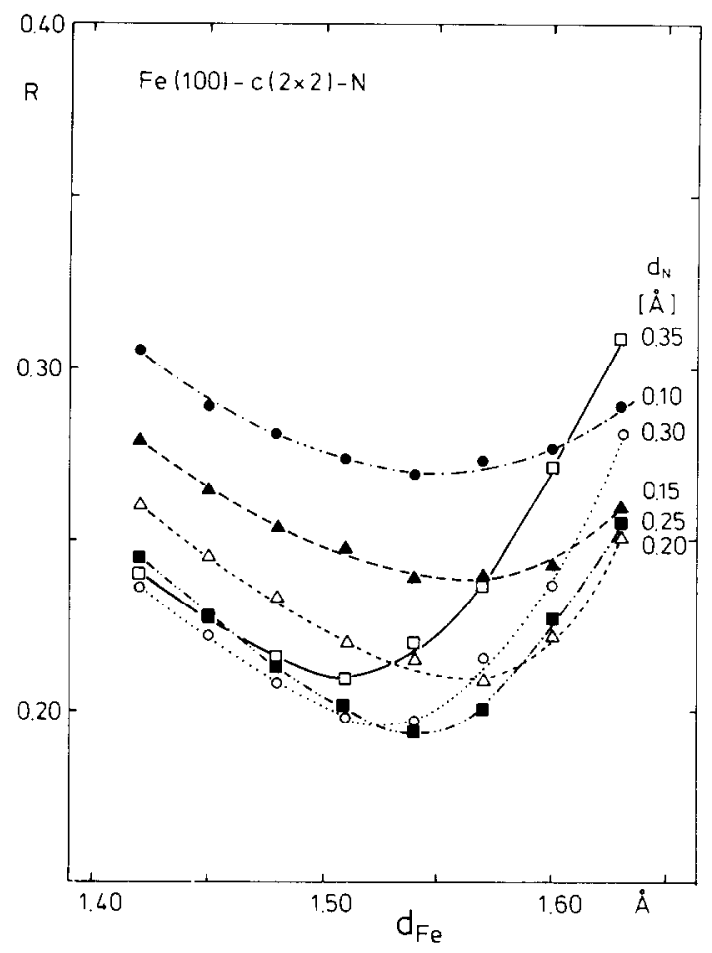

Fig. 8. $r$-Factor averaged over 16 beams ( 8 integral and 8 half-order) as a function of $d_{\mathrm{Fe}}$ at various values of $d_{N}$.

of $d_{\mathrm{N}}=0.27 \AA$ and $d_{\mathrm{Fe}}=1.54 \AA$ as sketched in fig. $3 \mathrm{~b}$. The distance between the topmost two Fe layers is thus increased by $0.11 \AA$ (=7.7\%) with respect to the bulk value. If compared with the clean $\mathrm{Fe}(100)$ surface, this spacing is expanded by $0.14 \AA(=10 \%)$ under the influence of the $\mathrm{N}$ overlayer. The bond length between the $\mathrm{N}$ atom and the underlying $\mathrm{Fe}$ atoms is $1.83 \AA$, while its distance to the four neighboring Fe atoms in the topmost layer is $2.04 \AA$. From the spread of the $r$-factor minima for the various beams and from the fact that the minima in fig. 8 are relatively flat, it is concluded that the indicated numbers are accurate to within $\pm 0.05 \AA$ as becomes evident from inspection of figs. $6-8$.

\section{Discussion}

To our knowledge so far only three papers concerning a LEED analysis of a surface containing nitrogen atoms have appeared in the literature: 
With the Ti(0001) surface (exhibiting completely different structure and symmetry than the system investigated here $)$ a $(1 \times 1)-\mathrm{N}$ structure was analysed in terms of penetration of nitrogen atoms below the topmost layer of $\mathrm{Ti}$ atoms [15]. The resulting structure is very similar to that of bulk TiN.

As with $\mathrm{Fe}(100)$, adsorbed $\mathrm{N}$ atoms form a $\mathrm{c}(2 \times 2)$ structure on the Mo(100) surface which was investigated by Ignatiev et al. [16]. In this work again on the occupation of fourfold sites was concluded, but the agreement between theory and experiment was not very satisfactory. The estimated distance between the nitrogen and the first metal layer $d_{\mathrm{N}}=1.0 \AA$ is considerably larger than that derived in the present work. The resulting $\mathrm{Mo}-\mathrm{N}$ bond length of $2.45 \AA$ appears to be unreasonably large. No expansion of the topmost two metal layers was allowed which would probably have improved the result.

Burkstrand et al. [17] studied the $c(2 \times 2)$ structure formed by $\mathrm{N}$ atoms on a $\mathrm{Cu}(100)$ surface by means of an averaging technique and concluded again on the occupation of fourfold sites. The interlayer distance $d_{N}=1.45 \AA$ appears again to be rather large (even in spite the fact that $\mathrm{Cu}-\mathrm{Cu}$ distance within the surface layer, $d_{x}^{\mathrm{Cu}}=2.55 \AA$, is smaller than in the case of iron, $d_{x}^{\mathrm{Fe}}=2.87 \AA$ ). A reexamination of the data suggested, however, that $d_{\mathrm{N}}$ is more likely around $0.8 \AA[18]$ which would correspond to a metal- $\mathrm{N}$ bond length of similar order as concluded in the present work.

As stated in the Introduction, the main motivation for the present study was the suggested similarity between the $F e(100)-c(2 \times 2)$ surface structure and the structure of bulk $\mathrm{Fe}_{4} \mathrm{~N}$ leading to the concept of "surface nitride". This assumption is nicely confirmed by the present results: $\mathrm{Fe}_{4} \mathrm{~N}$ crystallizes in the fcc lattice. In the (002) plane a $\mathrm{N}$ atom is coplanar with the four nearest $\mathrm{Fe}$ atoms $1.89 \AA$ apart and is directly above another $\mathrm{Fe}$ atoms at the same distance. Within the (002) plane of $\mathrm{Fe}_{4} \mathrm{~N}$ the nitrogen atoms form also a " $c(2 \times 2)$ "-structure. The local geometry of the adsorption site on the surface (fig. 3) differs in so far as the plane of the $\mathrm{N}$ atoms is $0.27 \AA$ above the plane of the four topmost $\mathrm{Fe}$ atoms. The distance of the $\mathrm{N}$ atom to the underlying $\mathrm{Fe}$ atom, $d_{1}$, is (within the limits of accuracy) identical to that in $\mathrm{Fe}_{4} \mathrm{~N}$, while the distance to the four Fe atoms in the surface layer is somewhat larger $\left(d_{2}=\right.$ $2.04 \AA$ ).

According to the structure model shown in fig. 3 and to the resulting values of $d_{1}$ and $d_{2}$ a substantial (if not the dominating) contribution of the bond formation arises from coupling of the $\mathrm{N}$ atom to the underlying $\mathrm{Fe}$ atom in the second atomic layer. The resulting atomic radius of the chemisorbed nitrogen atom $(0.59 \AA)$ is between the $N$ triple bond $(0.55 \AA)$ and double bond radius $(0.62 \AA)$. The strength of the $\mathrm{Fe}-\mathrm{N}$ bond (as derived from the adsorption energy) is about $140 \mathrm{kcal} / \mathrm{mol} \mathrm{[3]} \mathrm{and} \mathrm{thus} \mathrm{again} \mathrm{between} \mathrm{the} \mathrm{corresponding}$ values for the nitrogen triple bond $(225 \mathrm{kcal} / \mathrm{mole})$ and the double bond $(100$ $\mathrm{kcal} / \mathrm{mole}$ ) [19]. Thus a consistent picture for the structural and bonding properties of the $\mathrm{N} / \mathrm{Fe}(100)$ system is reached. 


\section{Acknowledgements}

The authors are grateful to Dr. V.L. Moruzzi for providing data on the iron and nitrogen potentials. Financial support of this work was obtained from the Deutsche Forschungsgemeinschaft (SFB 128).

\section{References}

[1] G. Ertl, Catalysis Rev. 21 (1980) 201.

[2] G. Ertl, M. Grunze and M. Weiss, J. Vacuum Sci. Technol. 13 (1976) 314.

[3] F. Bozso, G. Ertl, M. Grunze and M. Weiss, J. Catalysis 49 (1977) 18.

[4] F. Bozso, G. Ertl and M. Weiss, J. Catalysis 50 (1977) 519.

[5] K.H. Jack, Proc. Roy. Soc. (London) A208 (1951) 200.

[6] M. Grunze, F. Bozso, G. Ertl and M. Weiss, Appl. Surface Sci. 1 (1977) 103.

[7] G. Ertl, M. Huber and N. Thiele, Z. Naturforsch. 34a (1978) 30.

[8] K.O. Legg, F. Jona, D.W. Jepsen and P.M. Marcus, J. Phys. C10 (1977) 937.

[9] H.D. Shih, F. Jona, U. Bardi and P.M. Marcus, J. Phys. C13 (1980) 3801.

[10] H.D. Shih, F. Jona, D.W. Jepsen and P.M. Marcus, Surface Sci. 104 (1981) 33.

[11] V.L. Moruzzi, personal communication.

[12] J.B. Pendry, Low Energy Electron Diffraction (Academic Press, 1974).

[13] International Tables for X-ray Crystallography, Vol. III (Kynoch, Birmingham, 1962).

[14] E. Zanazzi and F. Jona, Surface Sci. 62 (1977) 61.

[15] H.D. Shih and F. Jona, Surface Sci. 60 (1976) 445.

[16] A. Ignatiev, F. Jona, D.W. Jepsen and P.M. Marcus, Surface Sci. 49 (1975) 189.

[17] J.M. Burkstrand, G.G. Kleiman, G.G. Tibbets and J.C. Tracy, J. Vacuum Sci. Technol. 13 (1976) 291.

[18] M.A. Van Hove, personal communication.

[19] G. Henrici-Olive and S. Olive, in: A Treatise on Dinitrogen Fixation, Eds. R.W.F. Hardy et al. (Wiley, 1979) p. 3. 